\title{
LES PRATIQUES DES APPRENANTS DU FRANÇAIS LANGUE ÉTRANGÈRE LORS DE LA RÉDACTION D'UN RÉSUMÉ DANS LE LMOOC PAROLES DE FLE
}

\author{
Practices among French-as-a-foreign-Language LeArners When Writing \\ a Summary in the PARoles de Fle LMOOC

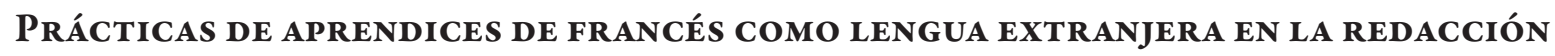 \\ DE UN RESUMEN EN EL LMOOC PAROLES DE FLE
}

\author{
Rana Hasan Kandeel \\ Docteure en sciences du langage, \\ Princess Nourah bint Abdulrahman \\ University. Professeure associée, \\ Department of Translation-French \\ program, Faculty of Languages, \\ Princess Nourah bint Abdulrahman \\ University, Saudi Arabia, Riyadh, \\ P. O. Box 84428 \\ RHKandeel@pnu.edu.sa
}

\section{Résumé}

Cette étude exploratoire à visée de recherche-action a pour objectif d'étudier l'effet de l'utilisation d'un LMOOC (language massive open online courses ou Language MOOC) sur les pratiques des apprenants FLE lors de la rédaction d'un résumé en français langue étrangère (FLE) par des étudiants jordaniens dans le département des langues modernes à l'université du Yarmouk. L'utilisation fait référence à l'acte d'utiliser les outils et les contenus tandis que l'usage renvoie à la manière habituelle de les utiliser. Pour réaliser cette étude, deux enquêtes ont été mises en place: une enquête consultative de type Delphi et une enquête qualitative de type entretiens. Les résultats ont montré que l'utilisation de ce cours en ligne pour rédiger un résumé introduit de nouvelles étapes dans l'activité rédactionnelle, telles que la prise de notes et l'évaluation par les pairs. L'utilisation des ressources externes et internes a eu un impact important non seulement sur les niveaux sémantique, graphomoteur et linguistique de l'activité rédactionnelle, mais elle développe également une attitude réflexive des étudiants vis-à-vis de leur écriture et de l'évaluation.

Mots-clés : language massive open online courses (LMOOC), français langue étrangère (FLE), pratiques de l'écriture académique, rédaction du résumé, ressources internes et externes.

\section{Abstract}

This sort of action-research exploratory study aims to examine the effect of an open and massive online language course (LMOOC, in English) on the practices of FLE learners in writing a summary in French as a foreign language (FLE, in French) by Jordanian students in the department of modern languages at Yarmouk university. To carry out our study, two surveys were implemented - a Delphi-type consultative survey and a qualitative interview-type survey. Results showed that the use of LMOOC in writing a summary incorporate new steps into writing activity, such as note-taking and review by peers. The use of external and internal resources has had an important impact not only on the semantic, graphomotor and linguistic levels of writing activity, but also on the students' reflective attitude towards their writing and peer assessment.

Reçu : 2018-03-10 / Accepté : 2018-08-27 / Publié : 2019-09-20

DOI : $10.17533 /$ udea.ikala.v24n03a10 
Keywords: language massive open online courses (LMOOC), French as a foreign language, practice of academic writing, summary writing, internal and external resources.

\section{Resumen}

El presente estudio exploratorio, con fines de investigación-acción, se propone analizar el efecto de un curso de lenguaje en línea abierto y masivo (LMOOC, en inglés) en las prácticas de los estudiantes de FLE al escribir un resumen en francés como idioma extranjero (FLE, en francés) por estudiantes jordanos en el departamento de lenguas modernas de la universidad Yarmouk. Para llevar a cabo el estudio, se implementaron dos instrumentos: una encuesta consultiva tipo Delphi y una encuesta cualitativa tipo entrevista. Los resultados mostraron que el uso de LMOOC en la escritura de un resumen incorpora nuevos pasos en la actividad de escritura, como la toma de notas y la evaluación por pares. El uso de recursos externos e internos tiene un impacto importante no solo sobre los niveles semántico, gramatomotor y lingüísticos de la actividad de escritura, sino que también promueve una actitud reflexiva de los estudiantes hacia su escritura y la evaluación por pares.

Palabras clave: lengua cursos masivos abiertos en línea (LMOOC), francés como idioma extranjero, práctica de escritura académica, redacción de un resumen, recursos internos y externos. 


\section{Introduction}

Depuis quelques temps, des formations en français langue étrangère sont offertes sur des LMOOC; l'écriture académique y est placée au centre des activités proposées. Cette étude aborde l'utilisation du LMOOC Paroles de FLE lancé par l'université de Nantes via la plateforme FUN. Notre recherche s' intéresse plus précisément à l'effet de l'utilisation d'un LMOOC sur les pratiques des apprenants FLE lors de la rédaction d'un résumé, cette activité complexe de production verbale implique des traitements qui mobilisent des connaissances diversifiées, des processus et des outils de transformation de l'information (Piolat, 2004).

Notre étude sur les difficultés de l'écrit scientifique (Kandeel, 2013) a en effet montré que le processus de rédaction est plus complexe et plus difficile pour les étudiants jordaniens qui n'ont pas pratiqué l'écriture académique en licence et n'ont donc pas développé cette habileté. C'est pourquoi la pratique du résumé devrait être travaillée avant les études en master, afin d'habituer les étudiants aux genres de l'écriture académique. C'est dans cette logique que nous avons incité nos étudiants à s'intégrer dans un LMOOC en FLE pour apprendre à écrire un résumé.

Nous présentons ici tout d'abord les étapes de la rédaction du résumé à partir d'une vidéo, en $\mathrm{y}$ incluant des fondements théoriques sur l'activité rédactionnelle du résumé dans un LMOoc. Les travaux sur lesquels s'appuie notre recherche ont également été abordés dans ce cadre.

La deuxième partie de cet article est consacrée à la problématique de la recherche, à ses questions et au contexte de l'étude: après une description de la méthodologie mixte utilisée, nous présenterons les résultats de la recherche, suivis de la discussion.

\section{Cadre théorique}

Les étapes de la rédaction du résumé à partir d'une vidéo

La rédaction d'un résumé à partir d'une vidéo demande à la fois la gestion d'opérations intellectuelles et temporelles (Piolat, 2010) ainsi que la gestion simultanée des activités langagières de compréhension de la vidéo et de rédaction du texte écrit. Les recherches de Piolat $(2004,2010)$ étudiant ces deux activités lors de la prise de notes constituent les références fondamentales de notre recherche. Pour comprendre, l'apprenant doit construire une représentation pré-verbale intégrée et cohérente comprenant les principaux événements et actions auxquels se réfère la vidéo (Piolat, Barbier et Roussey, 2003). Cette représentation n'inclut pas forcément les mots entendus et peut comprendre d'autres informations, telles que les connaissances stockées en mémoire. Un apprenant de langue étrangère qui prend note des informations fait simultanément deux types de traitement du message oral. Il s'agit de traitements micro et macro-structuraux afin de découper le message en unités de sens et de supprimer les informations de détail.

Comme c'est le cas pour toute autre activité rédactionnelle, pour rédiger un résumé le rédacteur met en œuvre des étapes composées de trois processus essentiels: la formulation, l'exécution et le contrôle (Kellog, 1996 et 1998, cité par Piolat et al., 2003, p. 122). Chaque processus est à son tour articulé en étapes, représentées dans le Tableau 1.

Tableau 1 Les processus de l'activité rédactionnelle, selon Kellog.

\begin{tabular}{lll}
\hline La formulation & \multicolumn{1}{c}{ L'exécution } & Le contrôle \\
\hline La planification & Mise en œuvre de & La lecture \\
La traduction & l'activité graphomotrice & La révision \\
\hline
\end{tabular}

Le processus de formulation est constitué de la planification et de la traduction. La planification consiste à récupérer des idées stockées en mémoire et à décider de leur validité afin de les organiser. La traduction concerne l'utilisation des compétences linguistiques pour transformer les représentations élaborées pendant la compréhension en éléments verbaux écrits.

L'exécution est une activité graphomotrice qui consiste à mettre en œuvre des activités complexes d'écriture afin d'écrire correctement les formes 
des lettres, de les organiser et de les présenter selon les normes orthographiques propres à la langue écrite.

Dans le dernier processus, soit le contrôle, les étapes de la lecture et de la révision sont réalisées pour vérifier la qualité de la rédaction. La lecture permet d'examiner tous les niveaux linguistiques $\mathrm{du}$ texte que le rédacteur est en train de composer, de vérifier sa cohérence et la réalisation des buts fixés lors de la planification (Piolat et al., 2003). Quant à la dernière étape de révision, elle permet de modifier les erreurs relevées en relisant le texte rédigé.

\section{La rédaction du résumé dans un LMOOC : une activité d'écriture complexe}

Le terme LMOOC est une combinaison du mot anglais language et de l'acronyme Mooc. Sokolik (2014) le définit comme « un mélange éclectique de pratiques et d'outils visant à ce que les étudiants utilisent la langue cible d'une manière significative et authentique, à l'instar des meilleures pratiques d'enseignement/apprentissage des langues ${ }^{1} \gg$ (p. 20). Cette définition suppose que la consultation des ressources linguistiques peut se faire dans toutes les étapes de la rédaction. Le caractère d'ouverture du LMOOC offre à l'apprenant un accès gratuit et illimité aux ressources en ligne. Cependant, l'apprenant de langue étrangère doit être en mesure d'utiliser les ressources technologiques et de les gérer de façon autonome (Charlier, 2014). Des ressources comme les moteurs de recherche et les dictionnaires peuvent aider à mâ̂triser les niveaux sémantique et grapho-moteur du traitement de l'information. Un apprenant de FLE peut, par exemple, utiliser un moteur de recherche ou un dictionnaire en ligne dans les phases du traitement des représentations pré-verbales des idées à communiquer (niveau sémantique), c'est-à-dire

1 C'est nous qui traduisons: "The LMOoC, in imitating the best practices of language teaching and learning, is an eclectic mix of practices and tools aiming to engage students in the use of the target language in meaningful and authentic ways". quand il cherche à comprendre la signification des mots entendus avant de les transcrire et à vérifier s'ils sont correctement écrits (niveau grapho-moteur) (Piolat, 2004).

La complexité de la rédaction du résumé dans un LMOOC pour un apprenant FLE est due, d'une part, à la gestion de ces niveaux, car il doit consacrer davantage de ressources à des traitements non automatisables comme la planification et la révision, tout en développant constamment des procédures métacognitives de gestion de l'ensemble de son activité (Piolat, 2004).

D'autre part, quand l'écriture se fait avec l'ordinateur, la rédaction d'un texte exige de mobiliser davantage de ressources attentionnelles. Piolat et al. (2003) soulignent que la recherche et l'extraction des bribes de textes (couper-coller), dans le but de préparer des notes pour la rédaction d'un texte, sont des opérations plus coûteuses lorsqu'il s'agit d'un support informatique plutôt que d'un support traditionnel. Selon Crinon et Marin (2010), l'activité d'écriture consiste à « s'insérer dans un réseau d'interactions diverses, à définir des intentions et à anticiper les réactions de futurs lecteurs, à utiliser les textes qu'on a lus, à exercer une activité critique sur les textes des autres » (p. 86).

Les principes théoriques qui fondent l'activité rédactionnelle dans un LMOOC font partie du connectivisme et de la théorie constructiviste. Nommée aussi intelligence collective, selon Springer (2015), le connectivisme suppose que l'apprentissage est avant tout social, il n'est pas juste individuel. La langue constitue un répertoire d'expériences sociales pour signifier pragmatiquement et elle est utilisée pour communiquer et signifier. Elle s' inscrit dans une perspective complexe d'interconnexion sociale. Les principes du social et de la construction des connaissances dans l'interaction représentent des éléments fondamentaux dans l'apprentissage, selon la théorie de Vygotsky (Martínez, 1996, p. 16). L'apprentissage est donc un processus de construction permettant le développement cognitif et langagier de l'apprenant 
dans le cadre des interactions avec les autres apprenants.

Dans notre étude, les processus de rédaction exigent de mobiliser des stratégies cognitives et métacognitives. Les stratégies cognitives impliquent « une interaction avec la matière à l'étude, une manipulation mentale ou physique de cette matière et une application de techniques spécifiques à l'exécution d'une tâche d'apprentissage $^{2} \gg$. Elles concernent essentiellement la pratique de la langue, la réception et l'émission des messages, l'analyse et le raisonnement, et la création des structures. Les stratégies métacognitives concernent la centration des apprentissages, la planification et l'évaluation (Cyr, 1998, pp. 32-33). Les pratiques de la rédaction du résumé à partir d'une vidéo telles que la répétition, l'utilisation des ressources, le classement, la prise de notes, l'élaboration, le résumé, la traduction, le transfert de connaissances et l'inférence font partie de ces types de stratégies.

\section{La littérature scientifique}

Les études sur l'utilisation des Moocs en langues sont variées quant à leurs types et à leurs objectifs. Dans son étude descriptive sur l'utilisation du MOoc pour enseigner les langues, Sader Feghali (2016) a étudié les enjeux des moocs. Selon l'auteure, ces cours ne different pas d'autres outils et plateformes permettant d'apprendre une langue sans contraintes spatio-temporelles. Ils proposent des activités et des contenus diversifiés qui peuvent viser un enseignement de connaissances lexicales et grammaticales au moyen d'exercices de drills, ainsi que des compétences réceptives (compréhension orale et écrite) et/ou des compétences productives (production écrite) (Feghali, 2016). Bien que cette étude aborde les enjeux des LMOoc sur les différents plans pédagogique, économique, technique, humain et institutionnel il ne prend pas en compte les usages concrets et les pratiques des apprenants lors de la réalisation d'une activité

2 O'Malley et Chamot (1990), cité par Paul Cyr (1998). langagière. Dans la présente étude, nous porterons notre attention précisément sur ces usages et pratiques lors d'une activité langagière spécifique, à savoir la rédaction du résumé.

Une autre étude, menée par Bárcena, Read, Martín-Monje et Castrillo (2014), analyse la participation des étudiants dans le premier LMOOC en Espagne sur un enseignement d'anglais professionnel. L'accent y est mis sur la participation des étudiants aux modules, aux activités des pairs et à l'interaction dans ce cours, sur le taux d'abandon et de satisfaction. Les résultats de cette étude ont montré que le taux d'abandon est important et nombreux sont les apprenants qui n'ont pas participé à tous les modules et aux activités des pairs. Malgré l'intérêt de ces résultats, cette étude de type quantitatif ne permet pas de comprendre la manière de mise en œuvre des activités langagières des apprenants. Les deux études évoquées ci-dessus s' inscrivent dans le cadre de l'enseignement des langues, sans toutefois viser une activité langagière particulière, contrairement à notre recherche.

La littérature scientifique sur l'écriture académique dans un LMOOC n'est pas très riche, étant donné la nouveauté de l'objet de recherche. Il est cependant possible de la considérer comme un cas particulier des travaux portant sur l'écriture médiée. Elle prend alors sa place dans des environnements permettant le dépôt des documents, des textes à relire par les pairs, de la communication entre les participants et de l'intégration de l'apprenant dans une communauté de pratique (Dervin, Johansson et Mutta, 2007). Ce n'est qu'à partir de 2012 qu'on a vu l'apparition des Moocs en écriture (Sokolik, 2016). Selon les statistiques fournies en 2015 sur le nombre de Moocs disponibles, onze seulement ont été consacrés à l'écriture académique parmi les deux mille cours dans différents domaines (Sokolik, 2016).

Le premier LMOOC destinéà l'écriture académique est intitulé College Writing 2x: Principles of Written English. Ce cours a été conçu par un chercheur-praticien à l'université de Berkeley aux 
Etats-Unis. Sokolik (2016) évoque l'idée que le LMOOC peut constituer un environnement approprié à l'écriture académique dans la mesure où il encourage la construction des communautés de communication et d'échange, même à l'extérieur de leur contexte de travail, l'accès aux cours à distance et le travail dans un environnement moins stressant que celui de la classe. Par contre, les défis que le LMOOC doit relever se situent au niveau des contenus proposés, de la participation aux forums de discussion, des outils d'évaluation, de risque de plagiat et d'abandon de cours.

Bien que cette dernière étude présente un intérêt au niveau de l'observation des pratiques des apprenants lors de la réalisation d'une écriture académique $\mathrm{du}$ point de vue de l'enseignant-concepteur, elle n'aborde pas un genre précis de l'écriture académique. Notre recherche est en continuité avec les recherches sur l'écriture académique dans un LMOOC mais elle porte spécifiquement sur le résumé.

\section{2}

\section{Problématique de la recherche}

L'articulation entre les besoins en langues, les pratiques de l'écriture qui sont influencées par les spécificités du monde numérique et les modes d'accès au savoir, est devenue un facteur déterminant pour un enseignement efficace en didactique $\mathrm{du}$ français dans l'enseignement supérieur.

Pour certains chercheurs, les LMOOC ne constituent pas une innovation théorique (Mangenot, 2014) ni une innovation pédagogique (Sader Feghali, 2016; Boullier, 2014). La théorie du connectivisme qui sous-entend l'apprentissage dans un MOOC ne serait qu'un courant pédagogique ou une doctrine. Pour ces auteurs, elle n'offre pas un nouvel apport scientifique, comparée à d'autres théories telles que le constructivisme et le cognitivisme qui régissent l'apprentissage en ligne; la dimension sociale des apprentissages est déjà connue par ces théories et l'idée de la cognition partagée entre les hommes et les artefacts n'est pas nouvelle.
Les LMOOC ne sont pas non plus une innovation pédagogique dans la mesure où ils mettent en ligne des cours à visée de transmission de savoirs et des activités langagières comparables à celles qui sont présentées dans d'autres formations en ligne. Or, en dépit de ces points de vue, nous constatons que l'utilisation de LMOOC est effectivement un mode d'apprentissage des langues étrangères dans le contexte jordanien et nous souhaitons savoir si ces pratiques numériques émergentes renouvellent les connaissances sur l'écriture médiée.

La question de l'innovation n'est donc pas la seule à prendre en compte dans la réflexion didactique de l'enseignement/apprentissage des langues dans la formation en ligne. En effet, la didactique des langues tient à une approche systémique liée à son projet en tant que discipline ayant le but de proposer des moyens pour améliorer un processus de formation qui se définit essentiellement par sa complexité (Puren, 2001). Malgré la nouveauté de l'expérience d'apprentissage, le LMOoc peut servir de levier de changement au niveau des pratiques d'écriture du fait de la diversité d'usage que les apprenants font de ce type de cours lors d'une activité rédactionnelle. L'hypothèse principale dans la présente recherche est la suivante: l'usage de LMOOC a un impact sur les étapes de la rédaction du résumé et sur les pratiques rédactionnelles. A ce stade de notre recherche nous formulons cette question: quel est l'impact de l'usage d'un LMOOC par des apprenants FLE sur l'ordre et la manière dont ils mettent en œuvre les étapes de rédaction d'un résumé?

\section{Contexte de la recherche}

L'étude se déroule dans le département des langues modernes à l'université du Yarmouk où les étudiants reçoivent une formation traditionnelle en langue étrangère (français, allemand, italien, espagnol, etc.). Le programme de français s'étend sur quatre années et les étudiants obtiennent à la fin de leurs études une licence en langues modernes (Bachelor of modern languages, en anglais). Aucun 
cours ne propose un enseignement d'écriture en français langue académique. Les cours de production écrite portent la plupart sur l'enseignement/ apprentissage de la grammaire et de la compréhension écrite plutôt que sur la production écrite. L'inclusion d'une classe d'étudiantes jordaniennes de langue française de l'université du Yarmouk dans le LMOoc Paroles de FLE a été faite pour réaliser des tâches d'écriture académique telles que le résumé et le compte-rendu ; nous avons effectué la recherche auprès de cette population.

La tâche d'écriture est proposée dans le cadre du LMOOC Paroles de FLE offert par l'université de Nantes en France ${ }^{3}$ afin de développer les compétences langagières des apprenants FLE. Le LMOOC s'est déroulé pendant six semaines, du 2 novembre 2015 au 20 décembre 2015. Il avait pour objectif de développer la compréhension orale tout en travaillant simultanément sur l'écriture académique. À partir du visionnage d'une vidéo, les participants étaient invités à rédiger un résumé qui pouvait être ensuite corrigé par les pairs de façon anonyme.

\section{Méthodologie de la recherche}

Pour réaliser notre étude, nous avons mis en place deux méthodologies: une enquête consultative de type Delphi et une enquête qualitative de type entretiens. La première porte sur le classement chronologique des étapes de la rédaction, son objectif étant de valider les étapes évoquées dans notre cadre théorique et leur séquence. L'enquête est constituée d'une seule question demandant aux enseignants experts en FLE de classer

3 Le MOOC est proposé par l'initiative France université Numérique (Fun) du ministère français de l'Enseignement supérieur et de la Recherche disponible sur https:// www.france-universite-numerique-MOOC.fr/. Il s'agit d'une initiative mettant la création de MOOC au premier plan et ayant l'objectif de mettre en valeur leurs meilleurs enseignants des universités françaises et d'attirer les meilleurs étudiants, notamment en provenance de pays émergents. La plate-forme française FUN a été inaugurée en janvier 2014 avec 88000 inscriptions sur 25 cours en ligne (Compagnon, 2014). chronologiquement les six premières étapes de la rédaction d'un résumé à partir d'un document audiovisuel. Les réponses proposaient un total de dix étapes mais dont quelques unes ne pouvaient pas être classifiées dans les étapes respectives du résumé. Dans cette enquête, nous avons adopté des éléments essentiels de la technique Delphi (anonymat, rétroaction et l'extraction simultanée des données), visant à atteindre un consensus à partir d'opinions d'experts (Ducos, 1983). L'intérêt de cette technique réside justement dans le consensus qui permet de faire le point à propos d'un sujet déterminé sur la base de questions quantifiables.

La deuxième enquête était qualitative. Nous avons recueilli les données de l'étude en réalisant des entretiens qui visaient à comprendre les pratiques adoptées par les étudiantes dans l'usage de ces cours massifs pour la réalisation de l'activité de rédaction. Comme nous l'avons déjà mentionné, les études sur les LMOOC portent surtout sur l'étude globale du contexte de ces cours plutôt que sur une activité langagière comme la production écrite complexe. Cela peut être expliqué par le fait que les données sont en trop grande quantité à cause du nombre élevé des étudiants inscrits dans un MOOC, ce qui rend difficile l'analyse qualitative des pratiques d'apprentissage. Selon Roland (2014), la pédagogie, vu l'ampleur du dispositif à mettre en œuvre, est parfois laissée pour compte ou limitée à l'analyse statistique - toutefois essentielle - des big datas. La méthode de notre recherche est donc originale dans la mesure où elle tient compte des pratiques de chaque étudiante participant au LMOOC, ce qui permet une compréhension approfondie des pratiques.

\section{Les participants}

L'enquête consultative de type Delphi a été menée auprès de huit experts du domaine du FLE ${ }^{4}$, elle a été accompagnée d'une note explicative indiquant

4 L'accord des experts en FLE, qui sont des collègues enseignant le FLE, à inclure leurs réponses dans cette étude a été approuvé par leur réponse orale et par e-mail à notre 
le but de l'enquête et les délais de réponses accordés. Quant aux participants à l'enquête qualitative, il s'agit de neuf étudiantes qui finissaient leur deuxième année d'études de français ${ }^{6}$. En effet, ces étudiantes se sont inscrites dans le LMOOC et elles ont fait les tâches présentées dans ce cours. C'est la raison pour laquelle nous les avons choisies pour participer aux entretiens. Elles représentent aussi le nombre total des étudiants qui ont participé au LMOoc. Leurs niveaux linguistiques varient d'A2 à B1 selon le Cadre Européen Commun de Référence pour les langues; sept étudiantes avaient le niveau $\mathrm{A} 2$ et deux étudiantes avaient le niveau B1. Elles avaient entre 19 et 21 ans. Elles ont toutes l'arabe comme langue maternelle, le français est la deuxième langue étrangère apprise dans le contexte éducatif jordanien. L'anglais est la première langue étrangère.

\section{Recueil et analyse des données}

Les données du Delphi ont été recueillies début décembre 2016. Les entretiens ont été réalisés en décembre 2015 à la fin du LMOOC et leur durée a été de quinze à vingt minutes. Nous les avons enregistrés en arabe afin de permettre aux étudiantes une expression spontanée au sujet des usages du LMOoc et de leurs pratiques. Nous avons fait la transcription des entretiens en arabe, puis nous les avons traduits en français avant de commencer le traitement et l'analyse des données.

Les réponses du Delphi ont été analysées quantitativement par le logiciel Surveymonkey et elles ont été affichées sous deux formes - tableau et graphique- en temps réel. Mais, pour l'analyse des entretiens, nous avons privilégié une analyse

demande de participer au questionnaire et par leur participation à notre questionnaire en ligne.

5 Les participants à l'enquête sont des étudiantes inscrites à un cours de compréhension orale que nous avons donné dans le même semestre où elles ont suivi le LMOoc. Leur accord nous a été transmis oralement avant d'enregistrer leur entretien.

6 Les experts et les participants ont été informé au préalable de la garantie de leur anonymat. thématique du contenu des réponses des étudiantes représentant des catégories, définies en fonction des deux axes principaux qui ont guidé l'entretien, à savoir les usages que les apprenants du FLE font du LMOOC dans les étapes de la rédaction d'un résumé et les pratiques influencées par ces usages. Les usages ont été catégorisés en fonction des pratiques de l'activité d'écriture en ligne et des étapes de la rédaction. Les données ont été traitées manuellement selon des grilles d'analyse d'usage et des pratiques d'écriture dans les différentes étapes de la rédaction.

\section{Résultats de la recherche}

Nous présentons dans cette partie les résultats des deux enquêtes illustrées par des extraits des entretiens.

\section{Enquête consultative de type Delphi}

Les étapes de la rédaction sont mises en ordre par les enseignants selon leur importance dans la rédaction (Tableau 2). Le score le plus élevé montre que l'étape est plus importante.

Ces étapes apparaissent selon leur fréquence dans les réponses des experts, elles commencent par la prise de notes, qui précède l'activité de la rédaction du résumé à partir du document audiovisuel. Les autres étapes, telles que la planification, la lecture et la traduction, viennent ensuite, suivies des étapes de l'identification de l'auteur du document et, de la mise en œuvre de l'activité graphomotrice. La révision dans le processus du contrôle n'est apparue que dans la septième étape, mais nous allons l'intégrer, en raison de son importance dans l'activité rédactionnelle. En effet, dans la conception du questionnaire, nous avons prévu des réponses comprenant les étapes de la rédaction du résumé évoquées ci-dessus (cf. Les étapes du résumé) et d'autres réponses concernant les autres genres d'écriture académique comme la synthèse.

A l'exception de l'étape de la révision, toutes les autres étapes ont été validées par les experts. 
Tableau 2 Classement par les experts des étapes de la rédaction selon les scores obtenus dans l'enquête consultative.

\begin{tabular}{llc}
\hline \multicolumn{1}{c}{ Étapes à classer } & \multicolumn{1}{c}{ Classement des étapes par les experts } & Score \\
\hline - Traduction & 1- Prise des notes & $8,25 \%$ \\
- Montage des citations & 2- Planification & $7,63 \%$ \\
- Expression de l'avis personnel de & 3- Lecture & $6,25 \%$ \\
l'auteur & & \\
- Mise en œuvre de l'activité & 4- Traduction & $5,88 \%$ \\
graphomotrice & & \\
- Planification & 5- Identification de l'auteur du document & $5,50 \%$ \\
- Révision & 6- Mise en œuvre de l'activité graphomotrice & $5,38 \%$ \\
- Lecture & 7- Révision & $5,00 \%$ \\
- Prise de notes & 8- Montage des citations & $4,25 \%$ \\
- Identification de l'auteur du document & 9- Expression de l'avis personnel de l'auteur & $4,00 \%$ \\
- Présentation du document proposé & 10- Présentation du document proposé & $2,88 \%$ \\
\hline
\end{tabular}

D'ailleurs, l'étape de la lecture, qui apparaît dans le processus du contrôle à la fin de la rédaction dans notre cadre théorique, est inscrite avec les étapes de la planification et de la traduction dans le processus de la formulation.

Nous sommes partis de ces étapes pour effectuer l'analyse qualitative et avons ajouté l'évaluation par les pairs comme une étape intégrée au processus $\mathrm{du}$ contrôle de l'activité rédactionnelle.

\section{Enquête qualitative}

L'analyse des entretiens montre que les pratiques d'écriture du résumé sont diversifiées et changent selon l'usage du LMOoc dans chacune des étapes de la rédaction et dans l'activité de l'évaluation par les pairs.

\section{La prise de notes}

La vidéo a été utilisée plusieurs fois dans la prise de notes. Neuf étudiantes l'ont visionnée entre trois et dix fois. Toutefois cet usage avait pour objectif d'écouter les informations plutôt que de voir la vidéo. Les autres usages concernent l'utilisation des dictionnaires en ligne, pour traduire les mots du français en arabe, et l'utilisation du moteur de recherche
Google, pour faire une recherche thématique des informations sur le sujet du résumé.

Toutes les pratiques reliées à l'usage de la vidéo sont essentiellement en rapport avec l'activité de l'écoute pour comprendre les informations entendues. Le visionnage et le révisionnage ont été faits par les neuf étudiantes pour plusieurs raisons: premièrement, pour construire des représentations préverbales des informations entendues et pour mieux comprendre la vidéo car la plupart des étudiantes ont constaté que le niveau de langue de la vidéo était supérieur au leur. Trois étudiantes ont exprimé avoir voulu tout comprendre et six étudiantes ont dit avoir voulu bien saisir certains mots. Deuxièmement, la réécoute a constitué la base de l'écriture du résumé et elle les a aidées à apprendre la manière de construire les énoncés en français.

Pendant l'écoute, les neuf étudiantes ont eu recours à la traduction vers la langue arabe des mots incompréhensibles en français. Quatre étudiantes ont procédé à la prise de notes en français afin de pouvoir utiliser les informations notées dans l'étape ultérieure du travail; ensuite, elles ont traduit ces notes en arabe avant de les reproduire en français dans le résumé. L'étudiante E. 9. explique ainsi son approche de prise de notes: 
E. 9. 14 J'ai essayé d'écouter la vidéo, de résumer les idées, de bien l'écouter plusieurs fois, de comprendre de quoi elle parle et ensuite de noter les idées une par une, de faire un brouillon, d'écrire les idées et de les relier.

La prise de notes a permis de retenir les informations nécessaires à d'autres opérations, telle l'exécution de l'écrit.

\section{Laplanification}

Pour faire le plan du résumé, deux usages ont été mis en œuvre: le visionnage de la vidéo et l'accès au forum de discussion pour consulter les résumés socialisés en ligne. Ceux-ci sont des résumés rédigés par d'autres étudiants sur la plateforme et qui n'avaient pas encore été évalués par des pairs ou par des personnes expertes.

Ne connaissant pas la manière dont elles pouvaient faire la rédaction de ce genre d'écriture académique, trois étudiantes ont été obligées de passer par ce qu'on appelle l'étape de découverte et d'imitation de l'organisation linéaire des idées entendues dans la vidéo:

E. 8. 18 Alors que quand j'ai lu, j'ai vu qu'ils ont commencé à dire que le docteur avait dit ceci et cela et j'ai donc fait la même chose

\section{La traduction}

Sept étudiantes ont dit que, pour transformer les représentations préverbales en éléments écrits et trouver les expressions nécessaires à la rédaction, elles ont consulté des sites internet, notamment des sites littéraires, comme les sites des contes et des comptines. L'autre usage concerne la recherche sur Internet du même sujet.

La pratique la plus récurrente s'est avérée être l'emprunt des mots et des expressions adéquats, c'est-à-dire que les étudiantes ont tenté d'acquérir un nouveau lexique et de nouvelles formules pour exprimer les idées avec une langue compréhensible au lecteur. Ainsi, la lecture d'articles sur le sujet Smart phone a été une pratique présente dans la rédaction.

\section{La mise en auvre de l'activité graphomotrice}

Pour exécuter l'écriture, toutes les étudiantes ont fait usage du traducteur de Google, du dictionnaire en ligne « Reverso », du moteur de recherche Google, du visionnage de la vidéo et de la consultation des travaux socialisés :

- Traduction des mots et vérification de l'écriture des mots

Pour savoir le sens des mots utiles à la rédaction du résumé, les étudiantes ont utilisé des dictionnaires en ligne comme « Reverso ». Le traducteur «Google translate » a été employé comme outil d'aide pour traduire certains mots et le moteur de recherche Google a servi d'outil de vérification de l'orthographe des mots ou de la structure des phrases:

E. 3. 22 Je l'(Google') ai utilisé pour vérifier mon écriture. J'écrivais par exemple la phrase pour vérifier le sens et la structure. Je la tapais sur Google pour voir s'il y avait un manque (Accent, mot ou lettre) pour le rectifier.

- Classement et organisation des idées

Le choix et le classement des idées les plus importantes étaient essentiels :

E. 2. 2. Tout d'abord, j'ai pris des notes sur le sujet abordé (De quoi parle-t-il en général ?). Puis, j’ai séparé ces notes. Sur quoi porte chaque idée?... je l'ai résumée de manière un peu détaillée (de quoi s'agitil ?) et je l'ai rédigée.

L'enchaînement des phrases clés était l'étape la plus importante dans l'activité de rédaction. Quatre étudiantes ont su comment lier les idées et les phrases pour obtenir un ensemble cohérent, ce faisant elles ont appris les connecteurs logiques.

Les conseils de la tâche écrite dans la rubrique « lien utile » présentaient une aide méthodologique générale pour la rédaction, sans toutefois fournir des ressources pour remédier à ce type de difficultés. 
- Choix de la forme la plus courte dans l'expression

Le fait de ne pas être habituées à la rédaction du résumé a incité les étudiantes à utiliser la forme la plus brève dans l'expression d'une idée. Elles ont eu recours à un substantif ou à un verbe au lieu d'écrire une proposition très longue. Pour les idées qui sont difficiles à mettre en mots en français, la stratégie la plus adoptée a été d'importer des expressions et des fragments provenant d'un autre document support pour compléter les phrases dans le texte.

\section{La lecture et la révision}

Lors de la mise en œuvre de la rédaction, ces deux étapes n’ont pas été séparées par les étudiantes. Les usages ont été le visionnage de la vidéo et la consultation des résumées socialisés, cela afin de pouvoir relire leurs résumés et vérifier la pertinence linguistique et thématique de ceux-ci, toutes les étudiantes ont comparé leur propre langue dans l'expression écrite avec la langue utilisée par les locuteurs dans la vidéo. Elles voulaient par ce biais savoir comment une expression peut être formulée par un locuteur natif de langue française.

E. 2. 2. L'écriture ! Je ne sais pas si c'était correct ou pas ! Mais l'essentiel c'est qu'on pouvait s'exprimer et faire une comparaison entre ce que nous, on dit, et ce qu'ils disent dans la vidéo. On trouvait qu'il y avait des différences entre notre façon de dire et la leur

L'approche comparative a été aussi adoptée concernant les résumés rédigés par d'autres apprenants participant au LMOOC, dans le but de comprendre comment l'ensemble du résumé pouvait être présenté; cela a conduit parfois à une imitation des textes socialisés sur les forums de discussion

E. 7. 50 J'ai lu certains commentaires et vu comment ils les avaient écrits. J'ai vu qu'ils ont commencé par « bonjour » et c'est là que j’ai eu l'idée de le commencer par « Bonjour ».

Les activités de révision ont permis aux étudiantes de voir leurs propres erreurs avant de soumettre leurs résumés à l'évaluation, et de réfléchir sur les expressions de la langue et sur sa structure. Quelques étudiantes étaient capables de reconnaître leurs difficultés et n'ont pas hésité à en parler.

\section{L'évaluation par les pairs}

Le LMOOC permet aux étudiantes de déposer leurs résumés pour qu'ils soient évalués par les pairs et de prendre deux ou trois résumés à évaluer. Les pratiques des étudiantes se sont réparties entre l'évaluation de ces travaux et la réflexion sur les résumés, voire sur la démarche de l'évaluation. Les pairs ont donné des conseils pour vérifier les règles grammaticales qui avaient été déduites des ressources peu fiables. Ces remarques ont été reçues de manière positive car elles ont poussé les étudiantes à relever leurs propres erreurs et à réfléchir sur la langue en s'inspirant des pairs. Il s'agit d'une rétroaction pour reconnaître les pratiques fautives dans l'écriture, comme la traduction littérale des phrases ou le copier-coller d'autres textes sur internet:

E. 7.72 Ils (les pairs) m’ont envoyé quelque chose. J'avais copié deux phrases de Google comme elles l'étaient. Et ils m'ont dit que j'étais un petit peu sortie du contexte. Pas du sujet mais ce nétait pas la même chose ce qui montre qu'ils avaient bien lu.

Deux étudiantes ont évoqué l'adoption d'une démarche analytique des résumés rédigés en langue maternelle sur Internet afin de comprendre les composantes d'un résumé, de déduire les critères d'évaluation de ce genre et de les rendre transversaux dans l'évaluation du résumé en langue cible.

L'insuffisance d'informations fournies dans le feedback sur les points faibles ou forts de la rédaction, et parfois le fait de réduire l'évaluation à l'attribution d'une note, ont été désavantageux car les étudiantes n'ont pas pu savoir les raisons de l'obtention de cette note.

\section{Discussion}

À partir des résultats de cette étude, nous avons remarqué que l'utilisation du LMOOC dans la rédaction d'un résumé a impacté les étapes 
de l'activité rédactionnelle et les pratiques des apprenants.

\section{Étapes de rédaction impactées par l'utilisation de LMOOC}

Outre les étapes évoquées par Piolat et al. (2003), qui représentent les processus de l'activité rédactionnelle dans le cadre théorique, nous avons constaté le surgissement de nouvelles étapes, telles que la prise de notes et l'évaluation par les pairs (voir Tableau 3).

La prise de notes à partir de la vidéo est devenue une activité par laquelle doivent passer tous les apprenants FLE pour rédiger un résumé. Bien que la compréhension des vidéos et l'écriture en langue étrangère aient demandé des efforts cognitifs importants, les étudiantes ont réussi à aller jusqu'au bout à la fois dans l'activité de compréhension et dans l'activité rédactionnelle. En effet, les processus mis en œuvre lors de la rédaction ont été stimulés parce que la production finale a été mise à l'évaluation. Sinha, Jermann, Li et Dillenbourg (2014) ont remarqué que les étudiants qui regardent la vidéo d'un cours de MOOC dans le but d'être bien évalués procèdent à un traitement cognitif élevé des informations malgré la possibilité d'avoir une surcharge cognitive.

L'évaluation par les pairs, contrairement à la prise des notes, n'était pas obligatoire mais elle vient à la fin de l'activité rédactionnelle pour faire réfléchir les étudiantes sur leurs productions écrites et sur l'évaluation.

Nous avons aussi constaté que dans un LMOOC les étapes de lecture et de révision n'apparaissent pas à la fin de la rédaction LMOOC. En effet, en utilisant les dictionnaires en ligne et le moteur de recherche, les étudiantes ont procédé à la révision pendant les étapes de la prise de notes et de la mise en œuvre de l'activité graphomotrice qui ne sont pas les dernières étapes dans la rédaction dans un LMOoc. D'ailleurs, l'étape de « traduction » n'a pas eu le même objectif que celle illustrée dans les travaux de Piolat (2003, 2004, 2010). Il ne s'agit pas d'utiliser les compétences linguistiques pour transformer les représentations faites dans la compréhension en éléments verbaux écrits mais la traduction dans un LMOOC implique plutôt la recherche de sens des mots dans une autre langue.

\section{Pratiques de rédaction impactées par l'utilisation de LMOOC}

Les pratiques de la rédaction du résumé ont été modifiées grâce à l'utilisation des ressources variées, qui a révélé l'exigence d'efforts plus importants lors de la rédaction en termes de capacités attentionnelles associées aux opérations de bas niveau, c'est-à-dire en rapport avec le niveau linguistique du texte rédigé. L'usage du moteur de recherche Google pour la vérification de l'orthographe des mots ou de la structure des phrases vient combler la lacune manifestée par certains résumés

Tableau 3 Étapes de la rédaction du résumé impactées par le LMOOc.

\begin{tabular}{|c|c|c|}
\hline \multirow{7}{*}{$\begin{array}{l}\text { Étapes de la } \\
\text { rédaction }\end{array}$} & $\begin{array}{l}\text { Rédaction de la prise des notes } \\
\text { selon Piolat et al. (2003) }\end{array}$ & $\begin{array}{l}\text { Rédaction du résumé dans un LMоoc } \\
\text { selon les entretiens }\end{array}$ \\
\hline & La planification & La prise de notes \\
\hline & La traduction & La planification \\
\hline & $\begin{array}{l}\text { Mise en œuvre de l'activité } \\
\text { graphomotrice }\end{array}$ & La traduction \\
\hline & La lecture & La mise en œuvre de l'activité graphomotrice \\
\hline & La révision & La lecture et la révision \\
\hline & & L'évaluation par les pairs \\
\hline
\end{tabular}


quand les étudiantes n'avaient pas automatisé l'écriture des mots et ses aspects formels. Cette pratique a été parfois complétée par le copier-coller à partir des textes sources, utilisé pour montrer que les compétences d'écriture etaient bien développées dans la langue cible, ce qui serait une explication possible $\mathrm{du}$ recours au plagiat par les étudiants au niveau universitaire (Keck, 2006). Néanmoins, même si l'utilisation du moteur de recherche Google apporte des aides aux problèmes formels dans la rédaction (l'orthographe et la morphologie), il reste inutile à résoudre les problèmes de conception et de cohérence. D’ailleurs, il se révèle utile pour les apprenants d'un niveau linguistique élevé (C1 et C2), mais il l'est moins pour les niveaux inférieurs (O’Regan, Rivens Mompean et Desmet, 2010).

Le visionnage de la vidéo et la consultation des résumés socialisés ont représenté les usages des ressources internes. Le premier usage a été très fréquent dans la plupart des étapes de la rédaction; à savoir dans la prise des notes, la planification, la mise en œuvre de l'activité graphomotrice, la lecture et la révision. La consultation des résumés socialisés sur le forum a été plus fréquente dans ces trois dernières étapes. Ces deux usages ont permis d'apporter une aide pour mettre en œuvre les opérations de haut niveau, telles que le classement des idées, l'organisation du texte et le travail sur sa cohérence. Dans le cadre du LMOoc, la consultation des résumés socialisés a réduit l'interaction des étudiantes avec les autres apprenants au sujet de l'activité rédactionnelle ou sur le sujet du résumé LMOOC.

Certains chercheurs (Sokolik, 2016; Karsenti, 2013) se sont interrogés sur l'efficacité des MOOC en matière d'interaction car la plupart des étudiants n'interagit effectivement que très peu ou même pas du tout dans les lieux d'interaction tels les forums de discussion. Plusieurs explications sont possibles à ce propos: la première concerne l'absence de l'enseignant ou du tuteur car sa présence indirecte peut avoir une influence sur le déroulement stratégique de l'interaction (Vlad, Codleanu, Dervin et Vasile, 2009). La deuxième explication est en relation avec la mise à disposition des apprenants des résumés socialisés par d'autres apprenants, ce qui diminue la possibilité d'intervenir pour poser des questions sur la rédaction du résumé. La troisième explication serait liée à la réalisation de l'activité rédactionnelle: Warnock (2015) confirme que les étudiants répondent davantage aux activités d'écriture en ligne et dans une moindre mesure à un usage spécifique de la technologie.

De plus, les résumés socialisés sur le forum des discussions, diffuséssansêtre révisés par un enseignant, peuvent constituer des modèles erronés. Une étudiante a dit qu'elle a imitéle résumé de son collègue en écrivant « Bonjour » au début de son résumé. Si le LMOoc Parole de FLE a pris en considération l'évaluation des résumés des étudiants par les pairs, un travail d'accompagnement dans les phases de la planification et de la révision reste tout de même à faire. Vigner (2015) propose des pistes pour améliorer les pratiques d'écriture en FLE en signalant l'importance d'apporter aux apprenants des aides à la planification, la nécessité de fournir des modèles d'appui en L2 et enfin le travail de coécriture à envisager entre natifs et non-natifs, car le but final dans un cours d'écriture en ligne devrait être axé sur l'écriture et non sur l'apprentissage de l'utilisation des outils technologiques.

L'évaluation par les pairs est une autre pratique constatée au niveau de la réflexion sur les erreurs en langue étrangère, permettant à chacune des étudiantes d'assumer tantôt le rôle d'experte tantôt celui d'apprentie.

Les étudiantes expertes ont mené une réflexion critique sur la production écrite des pairs en prenant conscience de leurs erreurs et en s'entrainant à prendre du recul par rapport aux productions écrites.

Quant aux étudiantes apprenties, elles ont appris à découvrir les erreurs grammaticales et syntaxiques ainsi que le plagiat dans leurs propres productions écrites grâce aux commentaires et conseils reçus 
dans la rétroaction après la correction des productions. La retroaction des pairs aide les apprenants à déterminer les nécessités linguistiques de l'activité de production en question (Little, 1997) et à l'améliorer. Elle constitue donc un élément qui favorise l'acculturation à la pratique du résumé et la prise de conscience progressive des spécificités discursives de ce genre.

Un autre intérêt de l'évaluation par les pairs concerne la réflexion sur les critères d'évaluation. Les étudiantes se sont servies de méthodes analytiques de ce type d'écriture académique en les déduisant des mêmes genres disponibles dans leur langue maternelle et c'est dans ce cas qu'on a vu surgir une nouvelle pratique dans l'écriture. Les étudiantes ont respecté ces critères, mais l'enseignant doit les discuter avec les apprenants-évaluateurs pour vérifier la compréhension de ces critères.

Si l'évaluation par les pairs dans un LMOoc est avantageuse, on ne peut nier qu'elle peut poser problème au niveau didactique. Watters (2012) souligne que, dans un cours d'anglais langue seconde, les feedbacks des pairs dans les MOOC sont variables, ils sont ou bien trop longs ou bien très courts, ils sont mal formulés en anglais et lorsqu' ils sont fournis en d'autres langues, ils s'avèrent incompréhensibles puisqu' ils sont mal traduits par Google. Les retours du traducteur automatique peuvent comporter des erreurs grammaticales et des commentaires inappropriés.

Pour ces raisons, nous pensons que les critères d'évaluation doivent être révisés et validés en concertation avec un enseignant présent dans le LMOOC, cela afin de vérifier leurs qualités linguistiques et méthodologiques et d'encourager les étudiants à donner un feedback au lieu de se contenter de donner des notes à leurs pairs. Cette validation est avantageuse pour l'étudiant expert et l'étudiant apprenti: le premier peut être formé et outillé de procédés d'évaluation fiable et le fait de disposer de ces outils réduit son sentiment d'hésitation ou de manque de confiance à participer à l'évaluation, faute de compétence linguistique suffisante ou de formation à l'évaluation. Quant à l'étudiant apprenti, la validation par un enseignant peut garantir un feedback complet et qualitatif.

\section{Conclusion}

À la fin de notre étude, nous pouvons dire que l'usage de LMOOC modifie non seulement lesétapes de la rédaction mais aussi les pratiques des étudiantes. C'est ainsi que certaines propositions au niveau de l'ingénierie des cours massifs en ligne peuvent être avancées. La présence de l'enseignant constitue une première exigence pour l'organisation du déroulement de la tâche d'écriture et la stimulation de l'interaction, et cela afin d'éviter le regroupement des étudiants dans des petits groupes en dehors de ce cours. L'enseignant doit intervenir dans la définition des critères d'évaluation par les pairs et orienter les étudiants en ce qui concerne les types et la qualité du retour à fournir aux pairs.

La deuxième exigence est relative à la modalité de la mise en œuvre de la tâche. Une tâche collaborative permet mieux de profiter des spécificités de l'apprentissage dans ce cours (ouverture et interaction) et de mettre en contact des apprenants non natifs et natifs.

La troisième exigence concerne les types d'aides à proposer aux apprenants FLE dans la rédaction de ce genre académique. Nous jugeons qu'il est nécessaire de produire une démonstration interactive des méthodes de construction du texte académique, avec des exemples concrets de la façon de relier les phrases et les idées car, même s'il maîtrise les structures linguistiques de cette langue, l'apprenant FLE reste, selon notre étude, peu en mesure de travailler tout seul sur la cohérence du texte. Il faut aussi réfléchir à la manière que l'apprenant pourrait adopter pour profiter de l'expérience des enseignants et des tuteurs expérimentés dans l'écriture académique.

Pour conclure, nous pensons que cette recherche constitue un point de départ pour d'autres recherches sur l'enseignement de l'écriture en ligne donnant 
aux enseignants des éléments à prendre en considération afin de rendre les LMOOC plus inclusifs et plus accessibles.

\section{Références}

Bárcena, E., Read,T., Martín-Monje, E., et Castrillo, M. D. (2014). Analysing student participation in Foreign Language Moocs: a case study. Dans U. Cress et C. Kloos (Eds.), EMOoCs 2014, Proceedings of the European MOOC Stakeholder Summit 2014 (pp. 11-17). PAU Education. Disponible sur http://e-learning-teleformacion.blogspot.com/2014/07/ emoocs-2014-proceedings-european-mooc.html\#. WmcCA6hl_IU [Récupéré le 22/1/2018].

Boullier, D. (2014). mooc : en attendant l'innovation. Distance et Médiations des Savoirs, 6. Disponible sur https://dms.revues.org/685 [Récupéré le 20/12/2017].

Charlier, B. (2014). Les MOoc : une innovation à analyser. Distance et Médiations des Savoirs, 5. Disponible sur https://dms.revues.org/531 [Récupéré le 28/8/2016].

Compagnon, A. (2014). Moocs et vaches à lait. Le Débat, 180, 170-178.

Crinon, J. et Marin, B. (2010). Réviser à distance pour apprendre à écrire des textes narratifs. Revue Française de Linguistique Appliquée, XV(2), 85-99.

Cyr, C. et Germain, C. (1998). Les stratégies d'apprentissage. Paris : CLE International.

Dervin, F., Johansson, M. et Mutta, M. (2007). Écriture académique: collaboration multimodale et «constructions identitaires » en FLA. Synergies Europe, 2. Disponible sur http://gerflint.fr/Base/Europe2.pdf [Récupéré le 20/10/2017].

Ducos, G. (1983). Delphi et analyses d'interactions. Futureless, 71, 37-44.

Kandeel, R. (2013). Analyse des difficultés des étudiants jordaniens dans l'écrit scientifique universitaire : le cas du rapport de recherche dans une formation FLE. Voix Plurielles, 10(2). Disponible sur https://brock. scholarsportal.info/journals/voixplurielles/index [Récupéré le 4/2/2016].

Karsenti, T. (2013). Mooc : Révolution ou simple effet de mode? Revue Internationale des Technologies en Pédagogie Universitaire, 10(2). Disponible sur http:// www.ritpu.org/IMG/pdf/RITPU_v10_n02_06. pdf [Récupéré le 2/5/2017].
Keck, C. (2006). The use of paraphrase in summary writing: A comparison of L1 and L2 writers. Journal of Second Language Writing, 15(4), 261-278.

Little, D. (1997). La compétence stratégique examinée par rapport à la maîtrise stratégique du processus d'apprentissage des langues. Dans H. Holec, D. Little, et R. Richterich (Eds.), Stratégies dans l'apprentissage et l'usage des langues : vers un Cadre Européen Commun de référence pour l'enseignement et l'apprentissage des langues vivantes : études préparatoires (pp. 9-40). Strasbourg : Conseil de l'Europe.

Mangenot, F. (2014). MOoc : hypothèses sur l'engouement pour un objet mal identifié. Distance et Médiations des Savoirs, 7. Disponible sur https://dms.revues. org/844\#tocto2n1 [Récupéré le 5/3/2016].

Martinez, P. (1996). La didactique des langues étrangères. Paris : Presses Universitaires de France.

O’Regan, B., Rivens Mompean, A. et Desmet, P. (2010). From spell, grammar and style checkers to writing aids for English and French as a foreign language: challenges and opportunities. Revue française de linguistique appliquée, $x V(2)$. Disponible sur https://www.cairn. info/revue-francaise-de-linguistique-appliquee-2010-2-page-67.htm [Récupéré le 7/4/2016].

Piolat, A., Barbier, M.-L. et Roussey, J.-Y. (2003). Mesure de l'effort cognitif : Pourquoi est-il opportun de comparer la prise de notes à la rédaction, l'apprentissage et la lecture de divers documents?Arob@se, 1-2. Disponible sur https://www.researchgate.net/ publication/228587788_Mesure_de_l'effort_cognitif_Pourquoi_estil_opportun_de_comparer_ la_prise_de_notes_a_la_redaction_l'apprentissage_et_la_lecture_de_divers_documents [Récupéré le $5 / 8 / 2016]$.

Piolat, A. (2004). Théories de l'écriture et pratiques scolaires. Approche cognitive de l'activité rédactionnelle et de son acquisition. Le rôle de la mémoire de travail. Linx, 54. Disponible sur https://linx.revues. org/174\#tocto1n2 [Récupéré le 9/9/2016].

Piolat, A. (2010). Approche cognitive de la prise de notes comme écriture de l'urgence et de la mémoire externe. Le français aujourd'hui, 170, 51-62.

Puren, C. (2001). La didactique des langues face à l'innovation technologique. Dans Abdi Kazeroni (dir.), Actes des Colloques Usages des Nouvelles Technologies et Enseignement des Langues Étrangères, II. Compiègne : Université Technologique de Compiègne, 1-13. Disponible sur http://www.utc.fr/ untele/ volume2.pdf [Récupéré le 25/12/2017]. 
Roland, N. (2014). De l'efficience de la production audiovisuelle pédagogique : entre considérations théoriques et analyses d'usages. Atelier présenté au colloque E-learning 3.0 : quel avenir pour la formation supérieur à l'ère du numérique. Université de Genève. Disponible sur http://mooc.hypotheses.org/131 [Récupéré le 5/10/2016].

Sader Feghali, L. (2016). Université 2.0. Un Mooc pour enseigner les langues? Disponible sur https://www. researchgate.net/publication/304624949_Universite_20_-_Un_MOOC_pour_enseigner_les_langues [Récupéré le 2/9/2017].

Sinha, T., Jermann, P., Li, N. et Dillenbourg, P. (2014). Your click decides your fate: Inferring information processing and attrition behavior from MOOC video clickstream interactions. Disponible sur http://arxiv. org/abs/1407.7131 [Récupéré le 22/7/2017].

Sokolik, M. (2014). What constitutes an effective language MOoc ? Dans E. Martín-Monje et E. Barcéna Madera (Dir.), Language Moocs: Providing learning, transcending boundaries (pp. 16-32). Berlin: De Gruyter Open LTD.

Sokolik, M. (2016). Academic writing in MOoc environments: Challenges and rewards. Dans E. Martín-Monje, I. Elorza et B. Riaza (Dir.), Technology-enhanced language learning for specialized domains. Practical applications and mobility (pp. 165-176). New York: Routledge.
Springer, C. (2015). Evaluation des compétences en langues : peut-on dépasser les standards ? Dans M. Watrelot, C. Polzin-Haumann (Dir.), L'évaluation des compétences langagières : un regard franco-allemand sur les défis et perspectives actuels (pp. 47-70). Namur : Presses Universitaires de Namur.

Vigner, G. (2015). L'écrit et la langue : Quelles priorités? Quelles articulations? Études de Linguistique Appliquée, 3(179), 269-280.

Vlad, M., Codleanu, M., Dervin, F. et Vasile, S. (2009). Types de remédiations dans la co-construction des discours en français langue académique : le cas de la communication médiée par ordinateur. Dans O. Galatanu, M. Pierrari et D. Raemdonck (Dir.), Construction du sens et acquisition de la signification linguistique dans l'interaction (pp. 237-251). Bruxelles : Peter Lang.

Warnock, S. (2015). Teaching the ow I course. Dans B. L. Heweth et K. DePew (Eds.), Foundational practices of online writing instruction (pp. 151-181). Colorado: The wac Clearinghouse. Disponible sur https:// ebookcentral.proquest.com/lib/pnbau-ebooks/reader.action?docID =3440287\&ppg $=1 \& q u e r y=$ mooc [Récupéré le 30/10/2016].

Watters, A. (2012). The problems with peer grading in Coursera. Disponible sur https://www.insidehighered.com/ blogs/hack-higher-education/problems-peer-grading-coursera. [Récupéré le 8/8/2017].

How to reference this article : Kandeel, Rana Hasan (2019). Les pratiques des apprenants du français langue étrangère lors de la rédaction d'un résumé dans le LMOOC Paroles de FLE. Íkala, Revista de Lenguaje y Cultura, 24(3), 487-502. DOI : 10.17533/udea.ikala.v24n03a10 